\title{
Increased Efficacy and Tolerability of Pegloticase in Patients With Uncontrolled Gout Co-Treated With Methotrexate: A Retrospective Study
}

John A. Albert · Tony Hosey · Brian LaMoreaux

Received: May 27, 2020 / Published online: July 27, 2020

(C) The Author(s) 2020

\section{ABSTRACT}

Introduction: Gout is a painful inflammatory condition caused by chronically elevated serum uric acid levels (sUA). When standard uratelowering therapies fail/are not tolerated, uncontrolled gout (elevated sUA, subcutaneous tophi, chronic gouty arthritis, frequent flares) can occur. Pegloticase, a recombinant uricase, converts uric acid to allantoin, a readily excreted molecule. Responder rate in trials was $42 \%$, limited by anti-drug antibody (ADA) development. Immunomodulators attenuate ADA formation and case reports suggest immunomodulation increases pegloticase responder rates. The current study retrospectively examined responder rate in patients undergoing methotrexate/pegloticase co-therapy.

Methods: Patients who underwent methotrexate/pegloticase co-treatment at a single rheumatology practice were included. Demographics, clinical, treatment, and safety

Digital Features To view digital features for this article go to https://doi.org/10.6084/m9.figshare.12630698.

J. A. Albert ( $\square)$

Rheumatic Disease Center, 7080 N. Port

Washington Road, Milwaukee, WI, USA

e-mail: jalbertmd@gmail.com

T. Hosey · B. LaMoreaux

Horizon Therapeutics plc, 150 S. Saunders Road,

Lake Forest, IL, USA parameters were collected. The primary outcome was the proportion of responders $(\geq 12$ biweekly pegloticase infusions, sUA $<6 \mathrm{mg} / \mathrm{dl}$ just prior to infusion 12).

Results: Ten patients (nine men, $52.3 \pm 13.5$ years) with uncontrolled tophaceous gout (erosive damage, ulcerative tophi, frequent flares, gout-related hospitalizations) were included. Patients had failed allopurinol (100-300 mg) or febuxostat (40 mg) therapy (doses not increased because of intolerance, kidney concerns, noncompliance, or rapid tophi resolution requirement). Baseline sUA was $9.42 \pm 2.05 \mathrm{mg} / \mathrm{dl}$. Along with standard pre-infusion prophylaxis, nine patients received subcutaneous methotrexate $(25 \mathrm{mg} /$ week) initiated 14-35 days before pegloticase and one patient received oral methotrexate $(12.5 \mathrm{mg} /$ week) initiated 14 days after pegloticase. Eight patients (80\%) were responders, receiving $15.5 \pm 3.8$ infusions (range, 12-21) over $31.8 \pm 9.5$ weeks. One patient had efficacy loss with mild infusion reaction during infusion 4 and one patient was lost to follow-up after infusion 5. One patient reported one gout flare. No new safety concerns emerged.

Conclusions: Methotrexate/pegloticase cotherapy resulted in a higher responder rate than the established $42 \%$ with pegloticase alone. Therefore, methotrexate/pegloticase co-therapy may safely allow more patients to benefit from a full treatment course, likely through ADA attenuation. 


\section{PLAIN LANGUAGE SUMMARY}

Uncontrolled gout is a painful inflammatory condition caused by excess uric acid in the blood. When standard oral medicines used to lower uric acid do not work or cannot be taken, pegloticase is the only remaining treatment option. Unfortunately, less than half of patients respond to pegloticase for an adequate amount of time because their immune system develops antibodies against the medicine, causing the medicine to be quickly removed from the body preventing a durable or prolonged response. Methotrexate has been shown to limit or prevent this immune response in patients treated with biologic therapies for autoimmune diseases. The current study found that eight of ten patients (80\%) treated with both methotrexate and pegloticase responded to treatment (received 12 or more biweekly pegloticase doses and had low uric acid levels in their blood just before infusion 12). No new side effects or safety concerns were reported. In this retrospective study, methotrexate appeared to allow more patients to benefit from a full course of pegloticase therapy.

Keywords: Immunomodulation; Methotrexate; Pegloticase; Uncontrolled gout

\section{Key Summary Points}

\section{Why carry out this study?}

- Gout is a painful, inflammatory condition caused by persistently elevated serum uric acid levels. Oral medications to lower serum uric acid can fail, leaving pegloticase as the only treatment option for uncontrolled disease (frequent gout flares, tophi, and joint disease).

- Pegloticase converts uric acid to a form the body can easily excrete. However, some uncontrolled gout patients develop anti-drug antibodies to pegloticase, which causes a loss of efficacy and increases the risk for infusion reactions. Because of this, physicians have begun to use immunomodulatory medicines, such as methotrexate, to prevent this immune response.
- This study was done to see if patients treated with both pegloticase and methotrexate had better response rates than with pegloticase alone. Responder was defined as a $\geq 12$ biweekly pegloticase infusion therapy course with a serum uric acid level $<6 \mathrm{mg} / \mathrm{dL}$ just prior to infusion 12.

\section{What was learned from the study?}

- The response rate with methotrexate/ pegloticase co-therapy was $80 \%$, which was higher than the established $42 \%$ with pegloticase alone.

- Methotrexate immunomodulation with pegloticase therapy was well tolerated and may allow more patients with uncontrolled gout to benefit from a full treatment course.

\section{INTRODUCTION}

Gout is an inflammatory disease triggered by elevated serum uric acid levels (sUA). A 2015-2016 national health study found that 9.2 million Americans are affected by gout [1], up from an estimated 8.3 million in 2007-2008 [2]. Needle-like monosodium urate crystals form when sUA levels rise above its serum solubility limit $(6.8 \mathrm{mg} / \mathrm{dl})$. These crystals then deposit on bones, in joints, and in soft tissues (including organs and cartilage) [3] and result in chronic inflammation throughout the body. This inflammation persists, even between gout flares when many patients do not exhibit classic gout symptoms (high levels of pain, joint swelling and redness). Joint pain and bone erosion commonly occur in patients with urate deposition, particularly in patients with uncontrolled gout.

Maintaining sUA levels below $6 \mathrm{mg} / \mathrm{dl}$ reduces the number of gout flares [4] and can reduce the size and number of tophi [5]. As a result, this is the recommendation of both American [6] and European [7] guidelines for gout management. Recommended sUA levels are even lower 
for patients with severe gout [6-8] $(<5 \mathrm{mg} / \mathrm{dl}$ $[7,8])$, characterized by frequent gout flares, subcutaneous (visible) tophi, and chronic gouty arthritis. Unfortunately, some gout patients cannot tolerate or do not respond to traditional urate-lowering therapies (ULTs) [9], while others are non-compliant with their ULT medication [10]. In these patients, sUA levels remain over $6 \mathrm{mg} / \mathrm{dl}$ and urate deposition continues, resulting in continued flares, enlarging/persistent tophi, and ongoing erosive damage. Reducing sUA to within treatment target is of extreme importance to improve patient health and improve quality of life. Tophi and frequent gout flares have both been associated with decreased quality of life and disability in patients with controlled [11] and refractory [12] gout.

Pegloticase (pegylated recombinant uricase enzyme), a medication approved by the United States Food and Drug Administration (FDA) for uncontrolled gout, converts uric acid to readily excretable allantoin and is highly effective in lowering sUA [13]. In fact, pegloticase has been shown to rapidly resolve tophi in patients who respond to therapy (i.e., maintain sUA below $6 \mathrm{mg} / \mathrm{dl}$ ) [14]. However, the proportion of patients who maintained a therapeutic response (sUA $<6 \mathrm{mg} / \mathrm{dl}$ ) during treatment months 3 and 6 was only $42 \%$ [13]. Additionally, approximately one-quarter of patients experience an infusion reaction in the absence of preinfusion uric acid monitoring [13]. Studies have associated the development of anti-drug antibodies with both loss of efficacy (accelerated drug clearance) and infusion reactions [15-17]. Because pegloticase is both highly efficacious in therapy responders and there are very limited treatment options for gout that is refractory to standard ULTs, efforts have been made to attenuate anti-pegloticase antibody formation. A limited number of case reports in the literature suggest that pegloticase/immunomodulation co-therapy may minimize or prevent antidrug antibody formation and allow more patients to complete a full course of therapy [17-25]. However, published reports have only included a limited number of patients and varied in immunomodulatory agent and dosing parameters. The current case series retrospectively investigated the role of methotrexate co- treatment on pegloticase responder rates. Treatment parameters (e.g., number of infusions, treatment duration), number of gout flares, and therapy safety were also examined.

\section{METHODS}

This retrospective, chart review study was reviewed and approved by the Western Institutional Review Board (IRB; Puyallup, WA; registration number: IRB00000533). The IRB assigned the study exempt status, waiving the requirement of informed consent. All patients with uncontrolled gout who began pegloticase therapy ( $8 \mathrm{mg}$ every 2 weeks) between January 2017 and September 2019 and were co-treated with methotrexate were included. All patients were cared for by a single physician (JAA) at a community medical practice.

All data were retrospectively collected from the medical records and were recorded in a deidentified manner. Data were collected using a standardized form and included subject demographics, disease characteristics, comorbidities, serial sUA levels, methotrexate treatment details, and specific safety information (e.g., gout flare, infusion reaction, laboratory values). The main outcome measure was the proportion of therapeutic responders, defined as those patients fulfilling the following conditions: (1) had a pegloticase therapy course of at least 12 biweekly infusions and (2) had an sUA $<6 \mathrm{mg} /$ dl immediately prior to infusion 12 . Though not always considered a full course of therapy, 12 infusions was chosen for comparison to the length of treatment in the randomized clinical trials of pegloticase. The total number of pegloticase infusions administered, therapy duration, and the safety of using these medications in combination were also examined. Patients who discontinued methotrexate for any reason were still included and considered a responder if they met the outcome criteria.

\section{RESULTS}

A total of ten patients with uncontrolled gout were included (Table 1). Average subject age was 
Table 1 Subject demographic and clinical characteristics

\begin{tabular}{|c|c|c|c|c|c|c|c|}
\hline Patient & Gender & Age (years) & $\begin{array}{l}\text { BMI } \\
\left(\mathbf{k g} / \mathbf{m}^{2}\right)\end{array}$ & $\begin{array}{l}\text { Pre-therapy } \\
\text { sUA (mg/dl) }\end{array}$ & $\begin{array}{l}\text { SubQ } \\
\text { tophi }\end{array}$ & $\begin{array}{l}\mathrm{eGFR}^{\mathrm{a}} \\
(\mathrm{ml} / \mathrm{min} / \\
\left.1.73 \mathrm{~m}^{2}\right)\end{array}$ & Comorbidities \\
\hline 1 & Male & 77 & 28.2 & 9.6 & Yes & 37 & $\begin{array}{l}\text { HTN, CKD, CAD, DM, } \\
\text { DL, lower lid mass }\end{array}$ \\
\hline 2 & Male & 61 & 27.9 & 9.0 & Yes & 91 & $\begin{array}{l}\text { DM, DL, OA, bipolar } \\
\text { disorder }\end{array}$ \\
\hline 3 & Male & 45 & 32.1 & 8.0 & Yes & 111 & HTN, anxiety \\
\hline 4 & Male & 64 & 26.9 & 5.7 & Yes & 59 & CKD \\
\hline 5 & Male & 57 & 25.2 & 8.1 & Yes & 60 & $\mathrm{OA}$ \\
\hline 6 & Male & 55 & 30.0 & 11.6 & Yes & 74 & $\begin{array}{c}\text { HTN, CKD, DM, OA, } \\
\text { DN, history of AKI }\end{array}$ \\
\hline 7 & Male & 29 & 22.9 & 9.5 & Yes & 106 & $\begin{array}{l}\text { HTN, anxiety, } \\
\text { polyarthralgia }\end{array}$ \\
\hline 8 & Female & 40 & 31.5 & 13.1 & Yes & 84 & $\begin{array}{l}\text { HTN, rheumatoid } \\
\text { arthritis }\end{array}$ \\
\hline 9 & Male & 49 & 30.2 & 10.6 & Yes & 76 & $\begin{array}{l}\text { HTN, OA, kidney } \\
\text { stone }\end{array}$ \\
\hline 10 & Male & 46 & 29.0 & 9.0 & Yes & 86 & $\begin{array}{l}\text { HTN, OA, } \\
\text { chondrocalcinosis } \\
\text { (wrist) }\end{array}$ \\
\hline Mean \pm SD & & $52.3 \pm 13.5$ & $28.4 \pm 2.8$ & $9.42 \pm 2.05$ & $10(100 \%)$ & $78.4 \pm 22.5$ & \\
\hline
\end{tabular}

$s U A$ serum uric acid levels; SubQ subcutaneous; $e G F R$ estimated glomerular filtration rate; $S D$ standard deviation; $H T N$ hypertension; $C A D$ coronary artery disease; $D M$ diabetes mellitus; $D L$ dyslipidemia; $O A$ osteoarthritis; $C K D$ chronic kidney disease; $D N$ diabetic neuropathy; $A K I$ acute kidney injury

${ }^{a}$ Calculated using serum creatinine levels using the abbreviated MDRD equation [23]

$52.3 \pm 13.5$ years and nine patients $(90 \%)$ were male. All patients had subcutaneous tophi as well as severe manifestations of gout, including numerous ulcerated tophi, recurrent gout flares, chronic pain, erosive damage on X-ray, or recurrent hospitalizations. These led to a markedly decreased quality of life in all patients and an inability to work in the majority of patients. Oral ULT use prior to pegloticase had included allopurinol (seven patients, $300 \mathrm{mg} /$ day; two patients, $100 \mathrm{mg} /$ day) and febuxostat (one patient, $40 \mathrm{mg} /$ day). Allopurinol/febuxostat doses were not increased to maximum doses because of drug intolerance, kidney concerns, patient noncompliance, and/or the need for rapid tophi resolution.

At the time of pegloticase therapy initiation, average sUA was $9.42 \pm 2.05 \mathrm{mg} / \mathrm{dl}$, with all patients having an sUA above target (range, $5.7-13.1 \mathrm{mg} / \mathrm{dl}$ ). Individual patient comorbidities are listed in Table 1 and included hypertension, coronary artery disease, diabetes, diabetic neuropathy, dyslipidemia, osteoarthritis, chronic kidney disease (CKD), kidney stones, and chondrocalcinosis of the wrist. All patients had at least one comorbidity, $80 \%$ had at least two comorbidities, and $60 \%$ had three or more comorbidities. Mean eGFR (calculated 


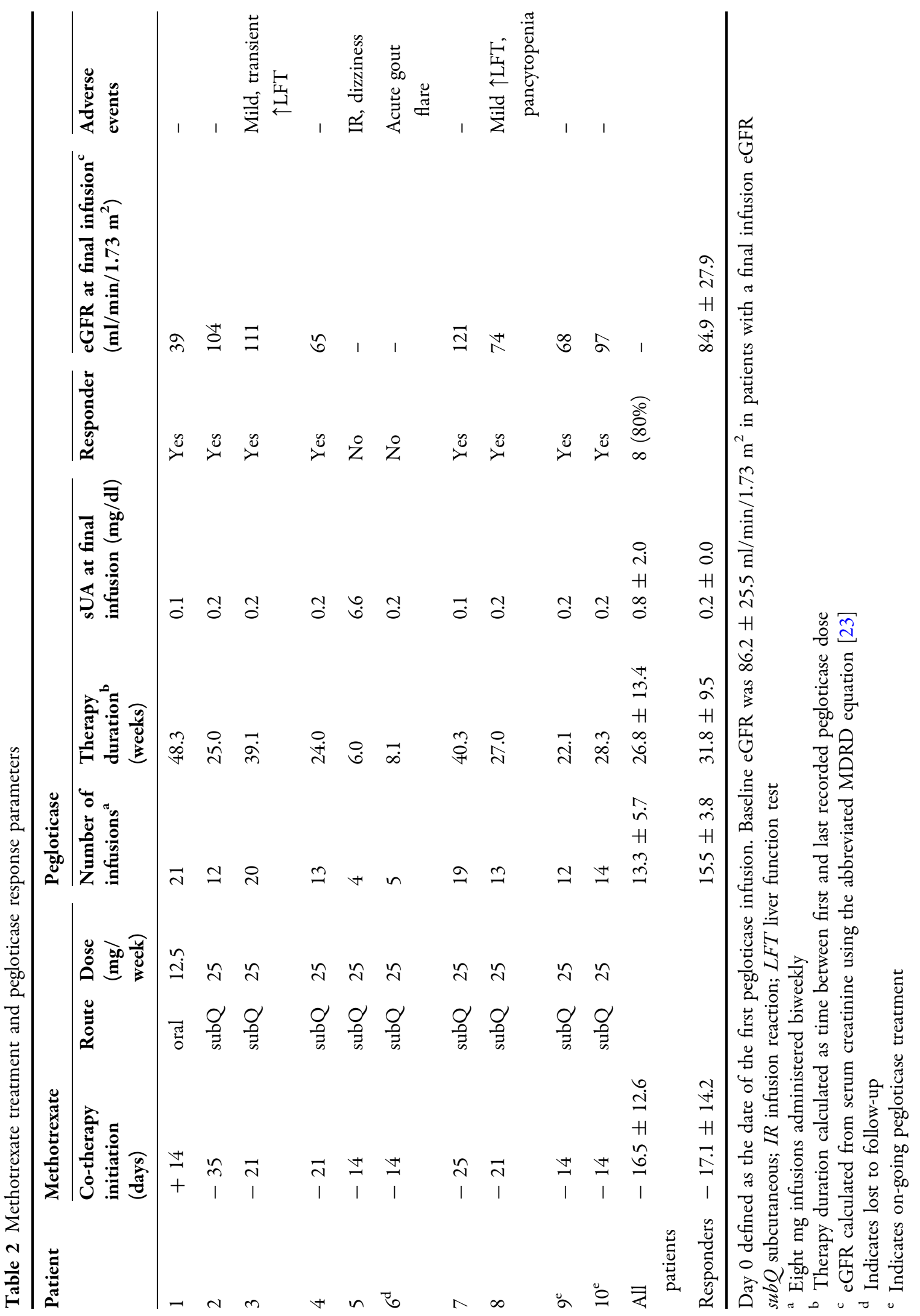



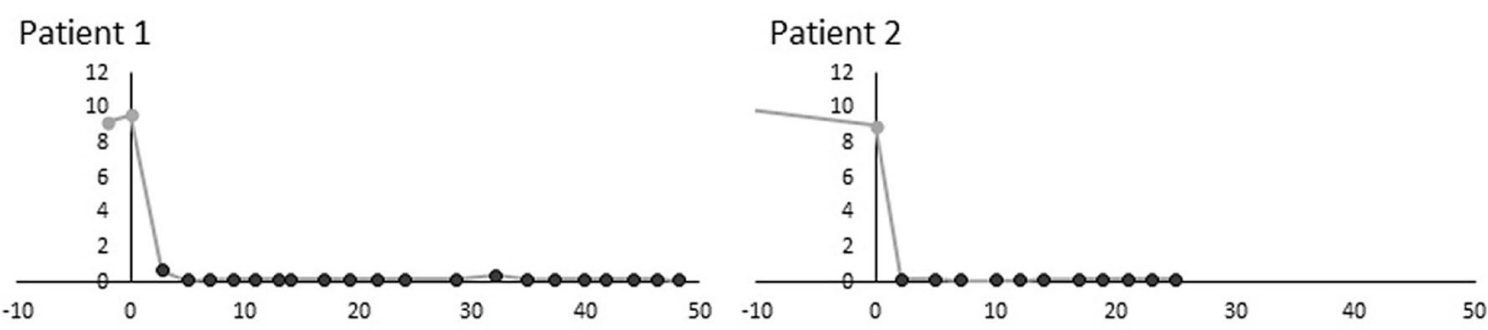

\section{Patient 3}
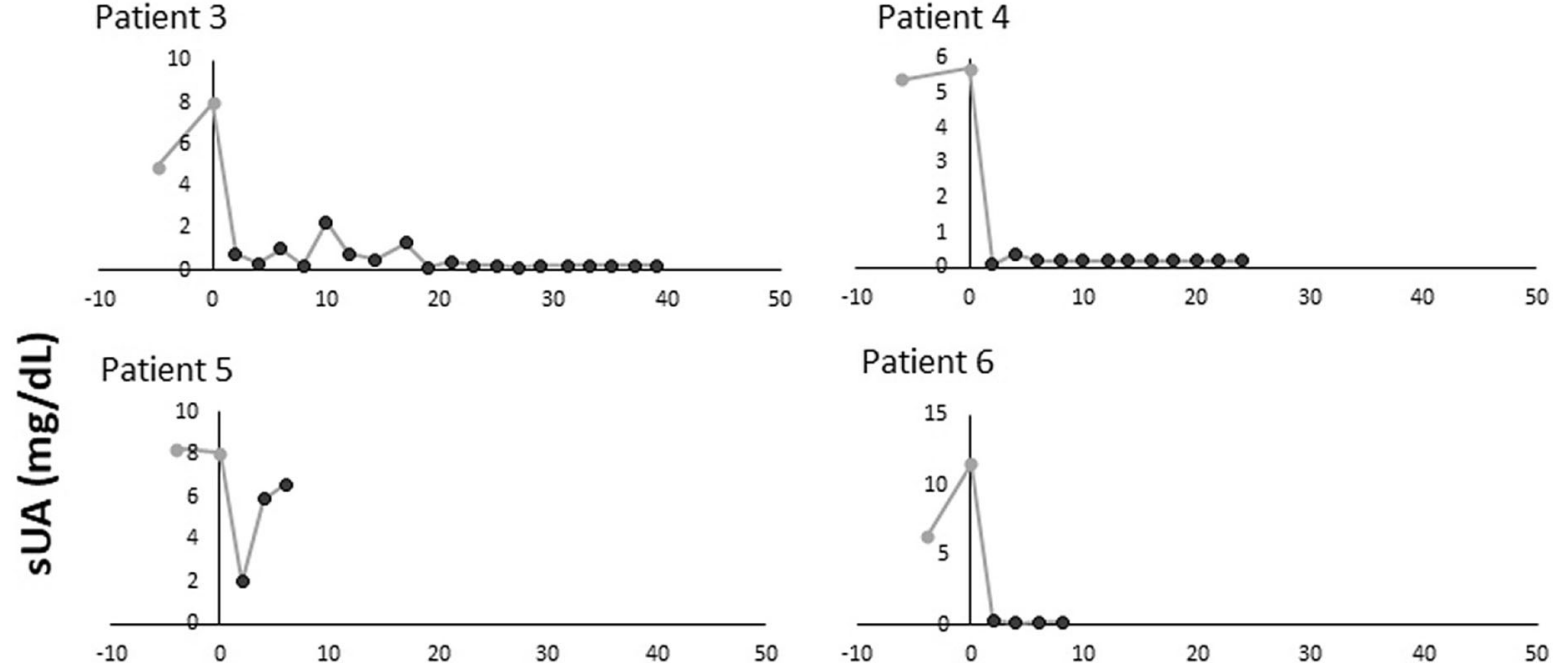

Patient 6
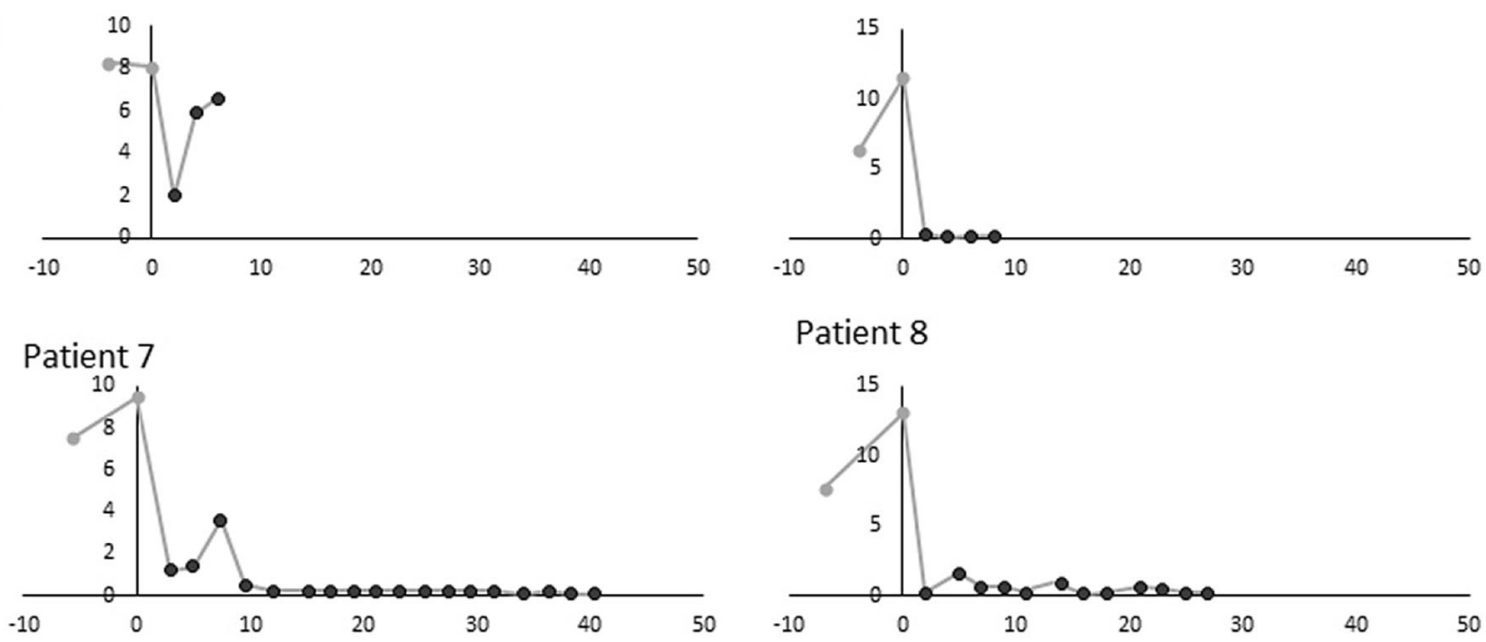

\section{Patient 8}
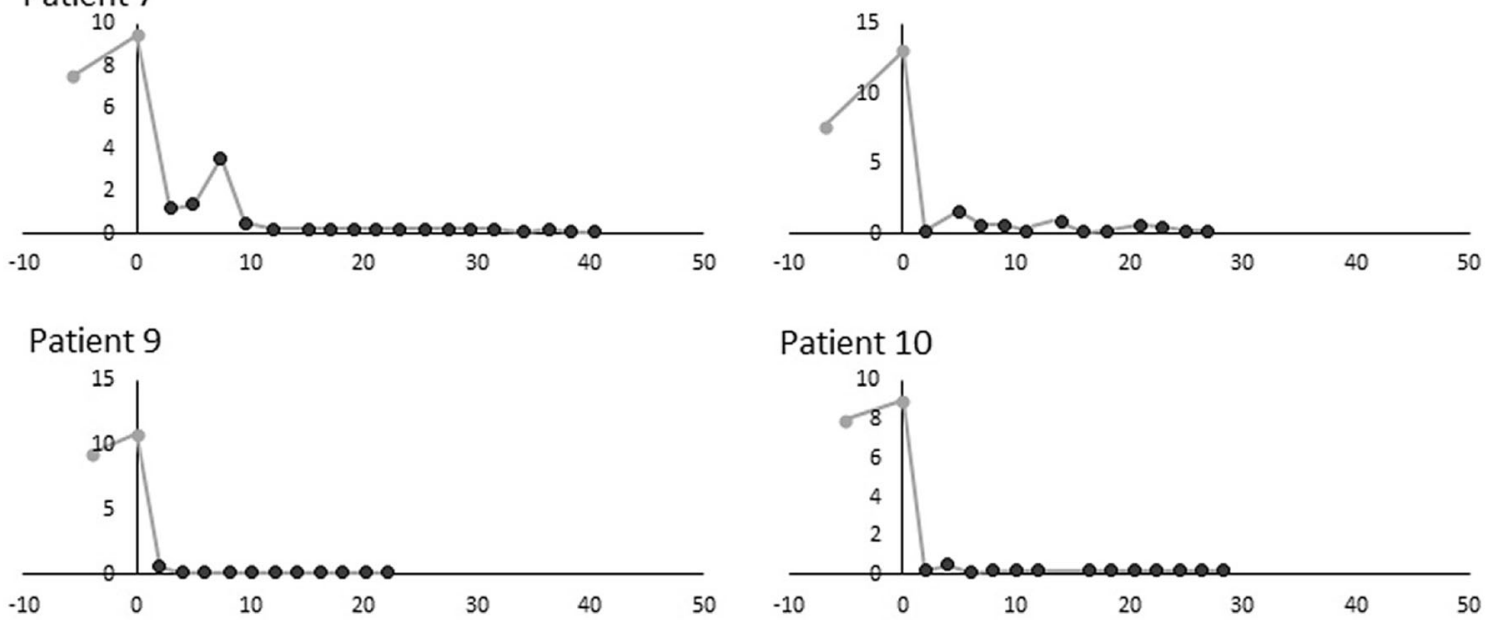

\section{Time (weeks)}

Fig. 1 Serial pre-infusion serum uric acid levels (sUA) in patients with uncontrolled gout who were co-treated with pegloticase and methotrexate. Day 0 was defined as the date of the first pegloticase infusion. Patients 5 and 6 were considered non-responders because of therapy discontinuation after infusion 4 (infusion reaction with sUA of $6.6 \mathrm{mg} / \mathrm{dl}$ ) and loss of follow-up after infusion 5 , respectively 
from serum creatinine levels [26]) was $78.4 \pm 22.5 \mathrm{ml} / \mathrm{min} / 1.73 \mathrm{~m}^{2}$. Per the practice's standard prophylactic infusion protocol, all patients were administered oral fexofenadine $(60 \mathrm{mg})$ the night before each pegloticase infusion and intravenous solumedrol $(125 \mathrm{mg})$ and oral fexofenadine $(60 \mathrm{mg})$ immediately prior to each infusion.

All patients were co-treated with pegloticase and methotrexate, as detailed in Table 2. Nine (90\%) patients began subcutaneous methotrexate $(25 \mathrm{mg} /$ week) an average of $19.9 \pm 7.0$ days prior to the first pegloticase infusion (range, 14-35 days before pegloticase). The remaining patient began oral methotrexate $(12.5 \mathrm{mg} /$ week) 14 days after beginning pegloticase therapy, just prior to the third pegloticase infusion. Once initiated, all patients were administered methotrexate on a weekly basis and daily oral folic acid ( $1 \mathrm{mg} /$ day) for the duration of pegloticase therapy. Eight of ten patients (80\%) were pegloticase responders, receiving at least 12 biweekly pegloticase infusions with an sUA below $6.0 \mathrm{mg} / \mathrm{dl}$ just prior to infusion 12 . All ten included patients had an initial, rapid decrease in sUA after initiating pegloticase therapy (Fig. 1). However, two patients stopped pegloticase therapy before receiving 12 infusions and were not considered responders. Patient 5 had a loss of response (pre-infusion sUA increased to $6.6 \mathrm{mg} / \mathrm{dl}$ ) in conjunction with a mild infusion reaction (skin rash, itching) during infusion 4. The patient was successfully treated with intravenous push anti-histamines (25 mg diphenhydramine $\mathrm{HCl}$ ) and oral glucocorticoids (10 mg prednisone at time of infusion reaction followed by $20 \mathrm{mg} /$ day for 5 days). Patient 6 , who was responding to therapy, experienced a gout flare on the day of infusion 5 . One week after infusion 5, this patient had a non-medical methotrexate injection issue and was lost to follow-up. The patient did not return for subsequent clinical follow-up or further pegloticase infusion.

No new safety concerns were identified. As stated above, one patient (Patient 6) reported a gout flare. One patient (Patient 5) had a loss of response (increased sUA levels) in conjunction with a mild infusion reaction (3-4 h duration; urticaria and erythema on face, neck, arms and trunk; dizziness). The infusion was stopped and the patient was administered $25 \mathrm{mg}$ intravenous diphenhydramine. All symptoms quickly resolved, with the exception of minor dizziness. Pegloticase therapy was discontinued in this patient.

All liver function tests (LFTs), blood counts, and laboratory values remained stable during therapy in all but two patients (Table 2). Patient 3 had a mild, transient increase in LFTs (ALT = $68 \mathrm{U} / \mathrm{L}$ ) prior to infusion 13 (coincident with alcohol consumption). The patient was advised to minimize alcohol use and the event resolved without intervention. The patient continued both methotrexate and pegloticase therapy, receiving an additional seven infusions without further issue and remained a treatment responder. Patient 8 did not disclose her alcohol use prior to therapy and had a mild, transient increase in LFTs (value not available; outside system) with concurrent pancytopenia. LFTs values returned to normal following methotrexate discontinuation and decreased alcohol consumption. Pancytopenia improved with brief hospitalization, red blood cell transfusion and methotrexate discontinuation. The patient received an additional four infusions of pegloticase (without methotrexate) without further adverse event and remained a responder throughout the treatment period. Kidney function remained stable (eGFR), with no patients developing new CKD or experiencing a worsening of existing CKD.

\section{DISCUSSION}

The current case series strongly suggests that immunomodulation therapy with methotrexate is effective in suppressing anti-drug antibody development in uncontrolled gout patients undergoing pegloticase therapy. In the current study, most patients began immunomodulation with subcutaneous methotrexate $(25 \mathrm{mg} /$ week) prior to the first pegloticase infusion, but one patient, who was a responder, began oral methotrexate $(12.5 \mathrm{mg} /$ week) 14 days after the first pegloticase infusion. Eight of ten patients $(80 \%)$ tolerated at least 12 biweekly pegloticase infusions and 
remained responders during their course of treatment (duration of $31.8 \pm 9.5$ weeks, $n=8$ responders). In contrast, only $42 \%$ of patients in the pegloticase clinical trials were treatment responders, with non-responders at a higher risk for ADA-induced infusion reactions [13]. These findings are of particular importance because pegloticase is the only FDA-approved therapy for uncontrolled gout.

Methotrexate and azathioprine have been shown to attenuate biologic anti-drug antibody formation in patients being treated for autoimmune diseases such as rheumatoid arthritis [27], spondyloarthropathies [28], and inflammatory bowel disease [29-31]. Oral methotrexate use with pegloticase has been previously reported in a limited number of cases [18, 21, 22, 24]. Bessen et al. $[18,22]$ reported a responder rate of $100 \%$ in six patients using methotrexate as the only immunomodulator. An additional responder was also reported in a patient who was initially administered methotrexate, but was switched to azathioprine because of ongoing fatigue [22]. In a series of ten cases [21] and an open-label clinical trial of 14 patients [24], response rates of $100 \%$ [21], and 79\% [24], respectively, were observed with oral methotrexate/pegloticase cotherapy (methotrexate initiated approximately 1 month prior to pegloticase in both studies). Some success has also been observed with other immunomodulators, including azathioprine [20, 25], leflunomide [23], mycophenolate mofetil [17, 19], and cyclosporine [17, 19, 22].

Our findings with oral and subcutaneous methotrexate immunomodulation are in agreement with studies and case reports in the literature. Adverse events reported included a gout flare in one patient and a mild infusion reaction associated with a loss of sUA response in another patient, both known effects of pegloticase. One patient experienced a mild, transient LFT elevation and pancytopenia, both known methotrexate side effects, and one patient experienced a mild, transient LFT elevation, which was deemed alcohol related.

This study had several limitations. First, the retrospective design may have introduced errors from a selection bias. Second, our conclusions are based on a limited number of patients and there was no direct comparison cohort.
Therefore, further prospective, controlled trials in a larger group of patients are needed to confirm the overall pegloticase responder rate when patients are co-treated with methotrexate and to determine the optimal immunomodulatory regimen. Such a trial to examine the efficacy and safety of oral methotrexate with pegloticase co-therapy is currently underway (MIRROR RCT, NCT03994731).

\section{CONCLUSIONS}

This case series demonstrated an $80 \%$ pegloticase response rate in patients with uncontrolled gout co-treated with methotrexate. Results presented here and in the literature suggest that immunomodulation co-therapy with methotrexate may allow more patients to be pegloticase responders with a favorable risk/benefit ratio.

\section{ACKNOWLEDGEMENTS}

Funding. Funds provided by Horizon Therapeutics plc (Lake Forest, IL) were used for thirdparty data collection fees and the journal's Rapid Service Fee.

Medical Writing, Editorial, and Other Assistance. The authors acknowledge Timothy Bailey, DO, PhD, CMPP (The Lockwood Group; Stamford, CT) for assistance with data collection and Lissa Padnick-Silver, PhD (Horizon Therapeutics; Lake Forest, IL) for assistance with data analysis and manuscript writing and editing.

Authorship. All named authors meet the International Committee of Medical Journal Editors (ICMJE) criteria for authorship for this article, take responsibility for the integrity of the work as a whole, and have given their approval for this version to be published.

Prior Presentation. Preliminary findings of this study were presented at the 2019 Annual Meeting of the American College of Rheumatology (November 8-13; Atlanta, GA). The 
complete analysis was presented at the 2020 European Congress on Rheumatology (June 3-6; Frankfurt, Germany).

Disclosures. John A. Albert has nothing to disclose. Brian LaMoreaux and Tony Hosey are employees of, and current stock and stock option holders in, Horizon Therapeutics plc.

Compliance with Ethics Guidelines. This retrospective, chart review study was reviewed and approved by the Western Institutional Review Board (IRB; Puyallup, WA; registration number: IRB00000533). The IRB assigned the study exempt status, waiving the requirement of informed consent.

Data Availability. The datasets generated during and/or analyzed during the current study are available from the corresponding author on reasonable request.

Open Access. This article is licensed under a Creative Commons Attribution-NonCommercial 4.0 International License, which permits any non-commercial use, sharing, adaptation, distribution and reproduction in any medium or format, as long as you give appropriate credit to the original author(s) and the source, provide a link to the Creative Commons licence, and indicate if changes were made. The images or other third party material in this article are included in the article's Creative Commons licence, unless indicated otherwise in a credit line to the material. If material is not included in the article's Creative Commons licence and your intended use is not permitted by statutory regulation or exceeds the permitted use, you will need to obtain permission directly from the copyright holder. To view a copy of this licence, visit http:// creativecommons.org/licenses/by-nc/4.0/.

\section{REFERENCES}

1. Singh G, Lingala B, Mithal A. Gout and hyperuricaemia in the USA: prevalence and trends. Rheumatology (Oxford). 2019;58:2177-80.
2. Zhu Y, Pandya BJ, Choi HK. Prevalence of gout and hyperuricemia in the US general population: the National Health and Nutrition Examination Survey 2007-2008. Arthritis Rheumatol. 2011;63:3136-41.

3. Perez-Ruiz F, Dalbeth N, Bardin T. A review of uric acid, crystal deposition disease, and gout. Adv Ther. 2015;32:31-41.

4. Shoji A, Yamanaka H, Kamatani N. A retrospective study of the relationship between serum urate level and recurrent attacks of gouty arthritis: evidence for reduction of recurrent gouty arthritis with antihyperuricemic therapy. Arthritis Rheumatol. 2004;51: 321-5.

5. Perez-Ruiz F, Calabozo M, Pijoan JI, Gerreri-Beites AM, Ruibal A. Effect of urate-lowering therapy on the velocity of size reduction of tophi in chronic gout. Arthritis Rheumatol. 2002;47:356-60.

6. Fitzgerald JD, Dalbeth N, Mikuls T, et al. 2020 American College of Rheumatology Guideline for the management of gout. Arthritis Care Res. 2020;72:744-60.

7. Richette P, Doherty M, Pascual E, et al. 2016 updated EULAR evidence-based recommendations for the management of gout. Ann Rheum Dis. 2017;76:29-422.

8. Pascart T, Latourte A, Flipo RM, et al. 2020 recommendations from the French Society of Rheumatology for the management of gout: urate-lowering therapy. Jt Bone Spine. 2020. https://doi.org/10. 1016/j.jbspin.2020.05.002.

9. Fels E, Sundy JS. Refractory gout: what is it and what to do about it? Curr Opin Rheumatol. 2008;20:198-202.

10. Löffler W, Fairbanks L. Refractory gout does it exist? Nucleosides Nucleotides Nucleic Acids. 2020. https://doi.org/10.1080/15257770.2020.1746804.

11. Khanna PP, Nuki G, Bardin T, et al. Tophi and frequent gout flares are associated with impairments of quality of life, productivity, and increased healthcare resource use: results from a cross-sectional survey. Health Qual Life Outcomes. 2012;10: 117.

12. Becker MA, Schumacher HR, Benjamin KL, et al. Quality of life and disability in patients with treatment-failure gout. J Rheumatol. 2009;36:1041-8.

13. Sundy JS, Baraf HS, Yood RA, et al. Efficacy and tolerability of pegloticase for the treatment of chronic gout in patients refractory to conventional treatment: two randomized controlled trials. JAMA. 2011;306:711-20. 
14. Mandell BF, Yeo AE, Lipsky PE. Tophus resolution in patients with chronic refractory gout who have persistent urate-lowering responses to pegloticase. Arthritis Res Ther. 2018;20:286.

15. Baraf HS, Yood RA, Ottery FD, Sundy JS, Becker MA. Infusion-related reactions with pegloticase, a recombinant uricase for the treatment of chronic gout refractory to conventional therapy. J Clin Rheumatol. 2014;20:427-32.

16. Lipsky PE, Calabrese LH, Kavanaugh A, et al. Pegloticase immunogenicity: the relationship between efficacy and antibody development in patients treated for refractory chronic gout. Arthritis Res Ther. 2014;16:R60.

17. Hershfield MS, Ganson NJ, Kelly SJ, Scarlett EL, Jaggers DA, Sundy JS. Induced and pre-existing antipolyethylene glycol antibody in a trial of every 3-week dosing of pegloticase for refractory gout, including in organ transplant recipients. Arthritis Res Ther. 2014;16:R63.

18. Bessen SY, Bessen MY, Yung CM. Recapture and improved outcome of pegloticase response with methotrexate-a report of two cases and review of the literature. Semin Arthritis Rheum. 2019;49: 56-61.

19. Freyne B. A case report of immunosuppressant medication-associated polyarticular tophaceous gout successfully treated using the polyethylene glycol-conjugated uricase enzyme pegloticase. Transpl Proceed. 2018;50:4099-101.

20. Berhanu AA, Krasnokutsky S, Keenan RT, Pillinger MH. Pegloticase failure and a possible solution: immunosuppression to prevent intolerance and inefficacy in patients with gout. Semin Arthritis Rheum. 2017;46:754-8.

21. Botson J, Peterson J. Pretreatment and co-administration with methotrexate improved durability of pegloticase response: a prospective observational, proof-of-concept, case series. Ann Rheum Dis. 2019;78:A1289.

22. Bessen MY, Bessen SY, Yung CM. Concomitant immunosuppressant use with pegloticase in patients with tophaceous gout: a case series. Int J Clin Rheumatol. 2019;14:238-45.
23. Masri K, Winterling K, LaMoreaux B. Leflunomide co-therapy with pegloticase in uncontrolled gout. Ann Rheum Dis. 2020;79(Suppl 1):450.

24. Botson J, Peloso PM, Obermeyer K, et al. Pegloticase response improvement by co-treatment with methotrexate: results from the MIRROR open-label clinical trial in patients with uncontrolled gout. Ann Rheum Dis. 2020;79(Suppl 1):442.

25. Rainey H, Baraf HSB, Yeo A, Lipsky P. Companion immunosuppression with azathioprine increases the frequency of persistent responsiveness to pegloticase in patients with chronic refractory gout. Ann Rheum Dis. 2020;79(Suppl 1):438.

26. Levey AS, Coresh J, Greene T, et al. Using standardized serum creatinine values in the modification of diet in renal disease study equation for estimating glomerular filtration rate. Ann Intern Med. 2006;145:247-54.

27. Krieckaert CL, Nurmohamed MT, Wolbink GJ. Methotrexate reduces immunogenicity in adalimumab treated rheumatoid arthritis patients in a dose dependent manner. Ann Rheum Dis. 2012;71: 1914-5.

28. Ducourau E, Rispens $\mathrm{T}$, Samain $\mathrm{M}$, et al. Methotrexate effect on immunogenicity and longterm maintenance of adalimumab in axial spondyloarthritis: a multicentric randomised trial. RMD Open. 2020. https://doi.org/10.1136/ rmdopen-2019-001047.

29. Ben-Horin S, Waterman M, Kopylov U, et al. Addition of an immunomodulator to infliximab therapy eliminates antidrug antibodies in serum and restores clinical response of patients with inflammatory bowel disease. Clin Gastroenterol Hepatol. 2013;11:444-7.

30. Strik AS, van den Brink GR, Ponsioen C, et al. Suppression of anti-drug antibodies to infliximab or adalimumab with the addition of an immunomodulator in patients with inflammatory bowel disease. Aliment Pharmacol Ther. 2017;45: 1128-34.

31. Jani M, Barton A, Warren RB, Griffiths CE, Chinoy $H$. The role of DMARDs in reducing the immunogenicity of TNF inhibitors in chronic inflammatory diseases. Rheumatology (Oxford). 2014;53:213-22. 